\title{
A Concept of Absolute Polarization in Dielectrics
}

\author{
Ishnath Pathak \\ Independent Researcher, Noida 201301, India
}

Received: December 08, 2016 / Accepted: December 19, 2016 / Published: April 30, 2017.

\begin{abstract}
It is enunciated in this paper that the volume density of the dipole moment of the induced charges in a dielectric does not in general qualify as a field in terms of which the actual induced charge distribution in the dielectric can be expressed as a volume charge density inside the interior of the dielectric equal to the negative of the divergence of that field and a surface charge density on the boundary of the dielectric equal to the component of that field in the direction of the outward normal to the boundary, unless the induced charge density inside the dielectric vanishes. The field that qualifies to satisfy the second criterion is in the general case named "absolute polarization", and the interconnection between the two polarizations is established. It is then demonstrated that although a few major equations of linear media electrostatics change, the results for the field of a uniformly polarized object remain unchanged, and all the existing methods of analytical evaluation can be justified if the "polarization" defined by the first criterion of being a field that equals the volume density of the dipole moment of bound charges is just replaced by the "absolute polarization", the concept of which is introduced here.
\end{abstract}

Key words: Forces and fields, conductors and dielectrics in constant electric field, non-uniform polarization fields.

\section{Introduction-The Meanings of and, Polarization}

What is actually meant when we talk about the polarization of a dielectric placed in an external electrostatic field? It is the vector field which at any point takes a value equal to the dipole moment per unit volume of the induced charges at that point in the dielectric. Consider two points $\vec{a}$ and $\vec{b}$ which can be chosen as origins for evaluating the dipole moment per unit volume. Let $\vec{r}$ be any general point in the dielectric. Let $R(V)$ denote a "physically infinitesimal" region of total volume $\mathrm{V}$ drawn around $\vec{r}$; the region can take all possible shapes. Let $\overrightarrow{P_{a}}(\vec{r})$ denote the macroscopic polarization at $\vec{r}$ when $\vec{a}$ is taken as the origin, and $\overrightarrow{P_{b}}(\overrightarrow{\mathrm{r}})$ be the same when $\vec{b}$ is taken as the origin. Now,

$$
\vec{P}_{a}(\vec{r})=\lim _{V \rightarrow 0}\left\{\left(\iint_{R(V)} \rho_{i}(\vec{r})(\vec{r}-\vec{a}) d \tau\right) / V\right\}
$$

Corresponding author: Ishnath Pathak, independent researcher, research fields: classical electromagnetics, optics, multivariable calculus. Email: pathak.ishnath@gmail.com.

$$
\vec{P}_{b}(\vec{r})=\lim _{V \rightarrow 0}\left\{\left(\iiint_{R(V)} \rho_{i}(\vec{r})(\vec{r}-\vec{b}) d \tau\right) / V\right\}
$$

where, $\rho_{i}(\vec{r})$ is the microscopic volume density of the induced charges in the dielectric at the point $\vec{r}$. Further,

$$
\begin{aligned}
& \vec{P}_{b}(\vec{r})=\lim _{V \rightarrow 0}\left\{\left(\iint_{R(V)} \rho_{i}(\vec{r})(\vec{r}-\vec{a}) d \tau\right) / V\right\} \\
& +(\vec{a}-\vec{b}) \lim _{V \rightarrow 0}\left\{\left(\iiint_{R(V)} \rho_{i}(\vec{r}) d \tau\right) / V\right\} \\
& =\vec{P}_{a}(\vec{r})+(\vec{a}-\vec{b}) \bar{\rho}_{i}(\vec{r})
\end{aligned}
$$

or, $\vec{P}_{b}(\vec{r})+\vec{b} \bar{\rho}_{i}(\vec{r})=\vec{P}_{a}(\vec{r})+\vec{a} \bar{\rho}_{i}(\vec{r})$, with $\bar{\rho}_{i}$ being

the macroscopic induced charge density at $\vec{r}$. Adding the term $-\vec{r} \bar{\rho}_{i}(\vec{r})$ to both sides, we get $\vec{P}_{b}(\vec{r})+\bar{\rho}_{i}(\vec{r})(\vec{b}-\vec{r})=\vec{P}_{a}(\vec{r})+\bar{\rho}_{i}(\vec{r})(\vec{a}-\vec{r}) \quad, \quad$ i.e. $\vec{P}_{b}(\vec{r})-\bar{\rho}_{i}(\vec{r})(\vec{r}-\vec{b})=\vec{P}_{a}(\vec{r})-\bar{\rho}_{i}(\vec{r})(\vec{r}-\vec{a})$. Thus we see, no matter which particular point $\vec{a}$ we intend to choose as the origin, the difference $\vec{P}_{a}(\vec{r})-\bar{\rho}_{i}(\vec{r})(\vec{r}-\vec{a})$ will be independent of $\vec{a}$ and 
will depend only on the point $\mathrm{P}$ in space which is labelled $\vec{r}$ by our coordinate system. We shall call this value as the value of the absolute polarization field at the point $\mathrm{P}$ and denote it as $\vec{I}_{e}(\vec{r})$. Everywhere in this paper we confine ourselves to static situations. So, a field is a scalar or vector function of positions in space and has no time-dependence. Similarly, when we say that a particular field is relative, we mean that its value at any position in space changes if we choose a different origin; an absolute field is one which doesnot change with a change of origin. Discussions in this paper do not involve relative motions of different reference frames. In terms of these terminologies, we see that $\overrightarrow{I_{e}}$ and $\bar{\rho}_{i}$ are absolute fields, whereas $\vec{P}$ is a relative field; and these fields are related by Eq. (1)

$$
\vec{P}=\vec{I}_{e}+\bar{\rho}_{i} \vec{r}
$$

Consider now the constitutive relation (Eq. (4.36) of Ref. [1]) for linear isotropic dielectrics: $\vec{P}=\varepsilon_{0} \chi_{e} \vec{E}$. Once a person becomes familiar with the preceding discussion, he would immediately render this equation incorrect. For $\vec{E}$ is an absolute field, while $\vec{P}$ is a relative field. So, if this equation is really incorrect, then we should have a nice reason for the fact that we did not face any difficulty in our works till now. Well, the reason is very simple: We used $-\vec{\nabla} \cdot\left(\varepsilon_{0} \chi_{e} \vec{E}\right)$ as the volume density of induced charges within the dielectric, and $\varepsilon_{0} \chi_{e} \vec{E} \cdot \hat{n}$ as the surface density of the induced charges on the boundary of the dielectric. This doing is right, the only mistake is $\varepsilon_{0} \chi_{e} \vec{E}$ is not $\vec{P}$ but $\overrightarrow{I_{e}}$. Let us consider this fundamental problem in detail. What physicists generally do (sections 4.1.4 and 4.2.1 of Ref. [2]) is that they define polarization $\vec{P}$ as the macroscopic dipole moment per unit volume of the induced charges and then prove a theorem that the actual macroscopic distribution of induced charges is a volume charge density $-\vec{\nabla} \cdot \vec{P}$ inside the region and a surface charge density $\vec{P} \cdot \hat{n}$ on the boundary. Let's at this very moment differentiate between these two fields. The first field - macroscopic volume density of the dipole moment of the induced charges - depends on the choice of origin, and in order to be completely specific, we shall denote this as $\vec{P}_{\wp}$, where $\wp$ is the point in space where the coordinate axes meet. The second field, being the solution to a well defined differential equation (which does not change with a change in the system of coordinates), subjected to an absolute boundary condition, is well defined and clearly does not depend on the choice of origin. We shall denote this field by $\overrightarrow{I_{e}}$. We have, of course, used this symbol previously to denote something else. The author has used the same symbol to avoid superfluity. If we used some other notation here, say $\vec{F}$ instead of $\overrightarrow{I_{e}}$, we would finally get $\vec{P}=\vec{F}+\overrightarrow{\rho_{l}} \vec{r}$, whence we argue $\vec{F}=\overrightarrow{I_{e}}$. We now proceed to find a relation between $\vec{P}_{\wp}$ and the field that we have denoted just now by $\overrightarrow{I_{e}}$. We evaluate $\vec{P}_{\wp}$ and $\overrightarrow{I_{e}}$ at a general location whose position vector w.r.t. origin $\wp$ is $\vec{r}$.

$$
\begin{aligned}
& \vec{P}_{\wp}(\vec{r})=\lim _{V \rightarrow 0}\left\{\left(\iint_{R(V)} \rho_{i}(\vec{r}) \vec{r} d \tau\right) / V\right\} \\
& =\lim _{V \rightarrow 0}\left\{\left(\iiint_{R(V)}\left(-\vec{\nabla} \cdot \underline{\vec{I}}_{e}\right) \vec{r} d \tau\right) / V\right\} \\
& \left(\vec{P}_{\wp}(\vec{r})\right)_{x}=\lim _{V \rightarrow 0}\left\{\left(\iint_{R(V)}\left(-\vec{\nabla} \cdot \vec{I}_{e}\right) x d \tau\right) / V\right\} \\
& =\lim _{V \rightarrow 0}\left\{\left(\iiint_{R(V)}\left(\vec{I}_{e} \cdot \hat{x}-\vec{\nabla} \cdot\left(x \underline{\vec{I}}_{e}\right)\right) d \tau\right) / V\right\} \\
& =\lim _{V \rightarrow 0}\left\{\left(\iiint_{R(V)} \underline{\underline{I}}_{e_{x}} d \tau\right) / V\right\}-\lim _{V \rightarrow 0}\left\{\left(\oiint_{S(V)} x \underline{\vec{I}}_{e} \cdot \vec{d} a\right) / V\right\} \\
& =\left(I_{e}\right)_{x}(\vec{r})-\lim _{V \rightarrow 0}\left(\frac{\bar{x}}{V} \oiint_{S(V)} \vec{I}_{e} \cdot \vec{d} a\right)
\end{aligned}
$$

where, $\vec{I}_{e}$ is the microscopic absolute polarization (while $\overrightarrow{I_{e}}$ is the macroscopic absolute polarization), and $\bar{x}$, according to a mean value theorem, is the $\mathrm{x}$-coordinate of some point in $R(V)$. Thus, 


$$
\begin{aligned}
& \left(\vec{P}_{\wp}(\vec{r})\right)_{x}=\left(I_{e}\right)_{x}(\vec{r})-\lim _{V \rightarrow 0}\left(\frac{\bar{x}}{V} \iiint_{R(V)}\left(\vec{\nabla} \cdot \vec{I}_{e}\right) d \tau\right) \\
& =\left(I_{e}\right)_{x}(\vec{r})-\lim _{V \rightarrow 0}\left(\frac{\bar{x}}{V} \iiint_{R(V)}\left(-\rho_{i}\right) d \tau\right) \\
& =\left(I_{e}\right)_{x}(\vec{r})+\left(\lim _{V \rightarrow 0} \bar{x}\right) \lim _{V \rightarrow 0}\left(\left(\iiint_{R(V)} \rho_{i} d \tau\right) / V\right) \\
& =\left(I_{e}\right)_{x}(\vec{r})+x \bar{\rho}_{i}(\vec{r})
\end{aligned}
$$

Hence, after gathering similar results for $\mathrm{y}$ and $\mathrm{z}$ components, we see $\vec{P}_{\wp}(\vec{r})=\vec{I}_{e}(\vec{r})+\bar{\rho}_{i}(\vec{r}) \vec{r}$. We shall call $\vec{P}_{\wp}(\vec{r})$ as relative polarization and $\vec{I}_{e}(\vec{r})$ as absolute polarization. To the author's knowledge, no one has distinguished between these two polarizations till now. In fact, no one till now has in this context ever meant by the term polarization anything other than what the author has called as relative polarization. In Section 3, we present a physical interpretation of absolute polarization, and to the author's knowledge no one has defined or described this physical quantity till now. Based on this physical interpretation of absolute polarization, we can write a mathematical expression for absolute polarization. Physicists often use this expression to derive the macroscopic equations from the microscopic Maxwell equations and they call the expression as polarization, despite that they themselves state the definition of polarization as that of relative polarization.

If a dielectric is placed in an external electric field, then (in terms of the total macroscopic electric field $\vec{E}$ due to all free and induced charges) the free charge density can be written $\bar{\rho}-\bar{\rho}_{i}$, i.e. $\varepsilon_{0} \vec{\nabla} \cdot \vec{E}+\vec{\nabla} \cdot \vec{I}_{e}$. Adhering to the same old notation we write this as $\vec{\nabla} \cdot \vec{D}$. We note here that we have not changed the definition of $\vec{D}$; i.e. even now we call as $\vec{D}$ what we used to call $\vec{D}$. Displacement is always defined (Eq. (57) of Ref. [3], section 4.3.1 of Ref. [4]) as that field (expressible as a combination of electric field and polarization) whose divergence resembles the free charge density to a proportionality constant typical of the units used. Only an expression for $\vec{D}$ changes:

$$
\vec{D}=\varepsilon_{0} \vec{E}+\vec{I}_{e}
$$

As $\vec{D}$ should come out to be equal to $\varepsilon_{0}\left(1+\chi_{e}\right) \vec{E}$, there results a small change in the appearance of the fundamental constitutive equation:

$$
\vec{I}_{e}=\varepsilon_{0} \chi_{e} \vec{E}
$$

\section{Field of a Uniformly Polarized Object}

One of the common problems in physics is to evaluate the field of a uniformly polarized object of polarization $\vec{P}$. There are two common ways (examples 4.2 and 4.3 of Ref. [5]) to tackle the situation. One is to argue that the field is due to a surface charge distribution on the boundary of the object varying as $P \cos \theta$, where $\theta$ is the angle between the direction of polarization and the outward normal to the surface. The other way is to picture the process of polarization as a small stretching of the object. It is said that there are two objects of the same shape and size, carrying uniform charge densities $+\rho$ and $-\rho$, which coincide when there is no polarization; and when polarization occurs, the geometrical centre of positive charge moves by a distance $d$ (in the direction of polarization) relative to the geometrical centre of negative charge. The field is evaluated as the net field of two uniformly charged objects in the limit $d \rightarrow 0, \rho \rightarrow \infty$ while the product $\rho d$ held fixed at the value $P$. In this section we'll see the justifications for these methods.

What is meant by a uniformly polarized object? With respect to which origin is the polarization uniform? Suppose that an object is placed in a weird electric field and the induced charge distribution inside its volume is an irregular and essentially non-uniform field $\bar{\rho}_{i}$. Assuming that it is possible, suppose that taking some point $\wp$ as the origin, the relative polarization $\vec{P}_{\wp}$ of the object comes out to be uniform. With respect to some other origin $\wp^{\prime}$, the relative polarization $\vec{P}_{\wp^{\prime}}$, being $\vec{P}_{\wp}+\bar{\rho}_{i} \vec{a}$, where $\vec{a}$ is the vector from $\wp^{\prime}$ to $\wp$, is a non-uniform field. 
So, we see that if polarization is uniform with respect to one origin, then it is not necessarily uniform with respect to another. By a uniformly polarized object we will mean that $\vec{P}$ is uniform with respect to all origins. So, for a uniformly polarized object we can form a first decision that the induced charge density $\bar{\rho}_{i}$ is uniform. But soon after that we notice that $\bar{\rho}_{i}$ must vanish. For $\bar{\rho}_{i}$, being $-\vec{\nabla} \cdot\left(\vec{P}-\bar{\rho}_{i} \vec{r}\right)$, must also equal $3 \bar{\rho}_{i}$.

Hence, we learn that for a uniformly polarized object, the relative polarization is the same with respect to all origins. This value, which also equals the absolute polarization, will be called the polarization of the object. Thus, for a uniformly polarized object of polarization $\vec{P}$, the distribution of induced charges is only on the surface and (as $\vec{P}$ equals $\overrightarrow{I_{e}}$ ) varies on the surface as $\vec{P} \cdot \hat{n}$ or $P \cos \theta$. Thus we now have a complete justification for our first method of evaluating the field of a uniformly polarized object. In fact our arguments were more like justifications for the validity of the problem itself. We now turn to justify the second method.

In a short note [6] published in 1992, David J. Griffiths introduced the concept that there exists a curious short method of solution of the field of a uniformly polarized object whenever it is easily possible to evaluate the field of a uniformly charged object of the same shape and size. He showed, as can be easily showed, that if the surface charge distribution over a closed surface is $\rho \vec{d} \cdot \hat{n}$ for two constants $\rho$ and $\vec{d}$ having the dimensions of volume charge density and length respectively, then its potential at any point in space is $\vec{E} \cdot \vec{d}$, where $\vec{E}$ is the electric field of a uniform volume charge $\rho$ within the region enclosed by the surface. For a surface charge distribution $\sigma=\sigma_{0} \cos \theta$ over a closed surface $\mathrm{S}$, with $\theta$ being the angle that the outward normal forms with the axis of $Z$, we can thus say that the potential $\Phi$ is given by $\sigma_{0} \overrightarrow{E_{1}} \cdot \hat{z}$, where $\overrightarrow{E_{1}}$ is the field per unit charge density due to a uniform charge distribution in the region enclosed by S. The field $\vec{E}$ of the surface charge is thus

$$
\begin{aligned}
& \vec{E}=-\vec{\nabla} \Phi=-\vec{\nabla}\left(\sigma_{0} \vec{E}_{1} \cdot \hat{z}\right)= \\
& -\sigma_{0}\left(\hat{z} \times\left(\vec{\nabla} \times \vec{E}_{1}\right)+(\hat{z} \cdot \vec{\nabla}) \vec{E}_{1}\right)=-\sigma_{0} \partial \vec{E}_{1} / \partial z
\end{aligned}
$$

The theorem just established has roots in an intuitive statement, and we shall call it as Griffith's normal charge theorem. It will be the licence to our second method of evaluation of the field of a uniformly polarized object.

Let's get back to what we do in our method. $\mathrm{S}$ is the boundary of a dielectric which encloses the region $\mathrm{R}$ occupied by the dielectric. $\mathrm{R}$ has an arbitrary shape. We now take an infinitesimally small distance $\Delta z$ and make another region $R^{\prime}$ by translating $\mathrm{R}$ in negative $\mathrm{z}$ direction by a shift of $\Delta z$. Now we fill R by a very large charge density $P / \Delta z$. We fill $R^{\prime}$ oppositely by a charge density $-P / \Delta z$. The combined system is neutral except for some places near the periphery of the regions $\mathrm{R}$ and $R^{\prime}$. Now, the field at any point $(x, y$, $z)$ due to the charge in $\mathrm{R}$ is $\left(\frac{P}{\Delta z}\right) \overrightarrow{E_{1}}(x, y, z)$, where $\overrightarrow{E_{1}}$ is the field per unit charge density due to a uniform charge distribution in $\mathrm{R}$. It is plain that the value of field per unit charge density at a general point $(x, y, z)$ due to a uniform charge distribution in region $R^{\prime}$ is the same as the value $\overrightarrow{E_{1}}(x, y, z+\Delta z)$. Hence, the field at any point $(x, y, z)$ due to the opposite charge in $R^{\prime}$ is $-\left(\frac{P}{\Delta z}\right) \overrightarrow{E_{1}}(x, y, z+\Delta z)$. So the net field of the combined system is $-P \frac{\vec{E}_{1}(x, y, z+\Delta z)-\vec{E}_{1}(x, y, z)}{\Delta z}$, i.e. $-P \partial \vec{E}_{1} / \partial z$. But according to our normal charge theorem this is the field due to a surface charge density $\sigma=P \cos \theta$ on $\mathrm{S}$. And, as we have already discussed in the first part of this section, this is exactly the field of the uniformly polarized object. Thus, we now have complete justifications for the two methods of evaluating the field of a uniformly polarized object that we described in the beginning of this section.

\section{Concluding Remarks}

In Section 1, the author claimed that physicists 
wrongly conclude that the actual distribution of induced charges in a dielectric is a volume charge density $-\vec{\nabla} \cdot \vec{P}$ inside the region and a surface charge density $\vec{P} \cdot \hat{n}$ on the boundary, with $\vec{P}$ being the relative polarization. The reason is very simple. When we claim that the dipole contribution at a point $\vec{r}$ in the multipole expansion of the potential of a configuration is $(\vec{p} \cdot \hat{r}) /\left(4 \pi \varepsilon_{0} r^{2}\right)$, it is assumed that $\vec{p}$ is the dipole moment of the configuration calculated with the same point (Eq. (4.8) and Eq. (4.10) of Ref. [7], Eq. (3.98) and Eq. (3.99) of Ref. [8]) as the origin from where the position vector of the point where the dipole contribution is being evaluated is $\vec{r}$. From the expression $\vec{P}=\vec{I}_{e}+\bar{\rho}_{i} \vec{r}$ connecting the relative and absolute polarizations, we see that the absolute polarization is the field which at any point in the dielectric takes a value equal to the dipole moment per unit volume at that point in the dielectric with the dipole moment being evaluated with that same variable point as the origin. This is why the potential of a dielectric at a point $\vec{r}$ outside the dielectric is $\left(1 / 4 \pi \varepsilon_{0}\right) \iiint\left(\vec{I}_{e}\left(\vec{r}^{\prime}\right) \cdot\left(\vec{r}-\vec{r}^{\prime}\right) /\left|\vec{r}-\vec{r}^{\prime}\right|^{3}\right) d \tau^{\prime} \quad$ and not $\left(1 / 4 \pi \varepsilon_{0}\right) \iiint\left(\vec{P}\left(\vec{r}^{\prime}\right) \cdot\left(\vec{r}-\vec{r}^{\prime}\right) /\left|\vec{r}-\vec{r}^{\prime}\right|^{3}\right) d \tau^{\prime}$.

Another remark is that $\vec{I}_{e}$ is defined only in the interior of a dielectric, and when I say that the surface density of induced charges is $\overrightarrow{I_{e}} \cdot \hat{n}$, it means that $\overrightarrow{I_{e}}$ there is the limit of $\overrightarrow{I_{e}}$ at that point on the surface from the interior of the dielectric. The net induced charge on the dielectric, being $-\iiint \vec{\nabla} \cdot \vec{I}_{e} d \tau+\oiint \vec{I}_{e} \cdot \vec{d} a$, is zero. An important remark is that the net dipole moment of the induced charges in the dielectric, which is an absolute quantity since the net induced charge on the dielectric is zero, is $\iiint \overrightarrow{I_{e}} \mathrm{~d} \tau$ where the integral is evaluated in the whole interior:

$$
\iiint \bar{\rho}_{i} \vec{r} d \tau+\oiint \bar{\sigma}_{i} \vec{r} d a=-\iiint \vec{r}\left(\vec{\nabla} \cdot \vec{I}_{e}\right) d \tau+\oiint \vec{r}\left(\vec{I}_{e} \cdot \vec{d} a\right) \quad,
$$

which

since

$\oiint \vec{r}\left(\vec{I}_{e} \cdot \vec{d} a\right)=\iiint \vec{r}\left(\vec{\nabla} \cdot \vec{I}_{e}\right) d \tau+\iiint\left(\vec{I}_{e} \cdot \vec{\nabla}\right) \vec{r} d \tau \quad, \quad$ equals

$\iiint\left(\vec{I}_{e} \cdot \vec{\nabla}\right) \vec{r} d \tau=\iiint \vec{I}_{e} d \tau$. Thus, the volume integral of the absolute polarization over the entire interior of a dielectric equals the total macroscopic induced dipole moment of the dielectric. Hence, if it's provided that this relation holds for a field $\overrightarrow{I_{e}}$, we cannot naively conclude that $\overrightarrow{I_{e}}$ is the relative polarization (see particularly Eq. (6.5) in section 6 of Ref. [9], where this mistake exists).

Yet another remark is that although $\iiint \rho_{i} d \tau=\iiint \bar{\rho}_{i} d \tau$ for all volumes, we cannot insist that $\iiint \rho_{i} \vec{r} d \tau=\iiint \bar{\rho}_{i} \vec{r} d \tau$ for all volumes, or what amounts to the same thing: $\vec{P} \equiv \bar{\rho}_{i} \vec{r}$, which is incorrect. For $\vec{P}$ can take a uniform value $\overrightarrow{P_{0}}$ inside the dielectric whereas whatever $\bar{\rho}_{i}$ may be and however it may vary, $\overline{\rho_{i}} \vec{r}$ cannot be uniform.

\section{References}

[1] Jackson, J. D. 1999. Classical Electrodynamics, 3rd edition. New York: Wiley, 154.

[2] Griffiths, D. J. 1999. Introduction to Electrodynamics, 3rd edition. NJ: Prentice Hall, 166-8.

[3] Purcell, E. M. 1965. Electricity and Magnetism, 1st edition. New York: McGraw-Hill, 332.

[4] Griffiths, D. J. 1999. Introduction to Electrodynamics, 3rd edition. NJ: Prentice Hall, 175.

[5] Griffiths, D. J. 1999. Introduction to Electrodynamics, 3rd edition. NJ: Prentice Hall, 168-169 and 172.

[6] Griffiths, D. J. 1992. "The Field of a Uniformly Polarized Object." American Journal of Physics 60 (2): 187.

[7] Jackson, J. D. 1999. Classical Electrodynamics, 3rd edition. New York: Wiley, 146.

[8] Griffiths, D. J. 1999. Introduction to Electrodynamics, 3rd edition. NJ: Prentice Hall, 149.

[9] Landau, L. D., Lifshitz, E. M., and Pitaevskii, L. P. 1984. Electrodynamics of Continuous Media, 2nd edition. Oxford: Elsevier Butterworth-Heinemann, 34-5. 